\title{
Mögliche Alternative: Blockade von nNOS
}

Schmerzen des Pankreas sind schwer behandelbar, da viele Schmerzmittel nicht wirken. Forscher der Technischen Universität München (TUM) haben nun den Grund dafür herausgefunden: Ein körpereigener Neurotransmitter liegt in den Nerven des Organs in sehr hohen Konzentrationen vor.

Im Bereich des Pankreaskopf ist die Nervendichte besonders hoch, daher kommt diesem bei der Schmerzentstehung und -wahrnehmung eine entscheidende Rolle zu. Auf der Grundlage von Gewebeproben aus dem Pankreaskopf von 42 Patienten, die an einer chronischen Pankreatitis oder einem Pankreaskarzinom litten und der Bestimmung der Menge der wichtigsten dort wirkenden Neurotransmitter, erstellte das Team um Priv.-Doz. DDr. Ihsan Ekin Demir ein Schmerzbotenstoffprofil für diesen Bereich der Bauchspeicheldrüse. Als
Kontrollen dienten Gewebespenden von gesunden Personen. "So lassen sich krankhafte Veränderungen gut erkennen", erklärt Demir. Dabei stellte sich heraus, dass die Stickstoffmonoxid-Synthetase (nNOS) in den Nerven des untersuchten Gewebes der Patienten stark erhöht war. Das Enzym ist für die Herstellung von Stickstoffmonoxid (NO) verantwortlich, das als Botenstoff unter anderem bei der Schmerzbildung eine Rolle spielt. Insbesondere führt NO über die Bindung an seine Rezeptoren auf der Neuronenoberfläche zu einer Überaktivierung

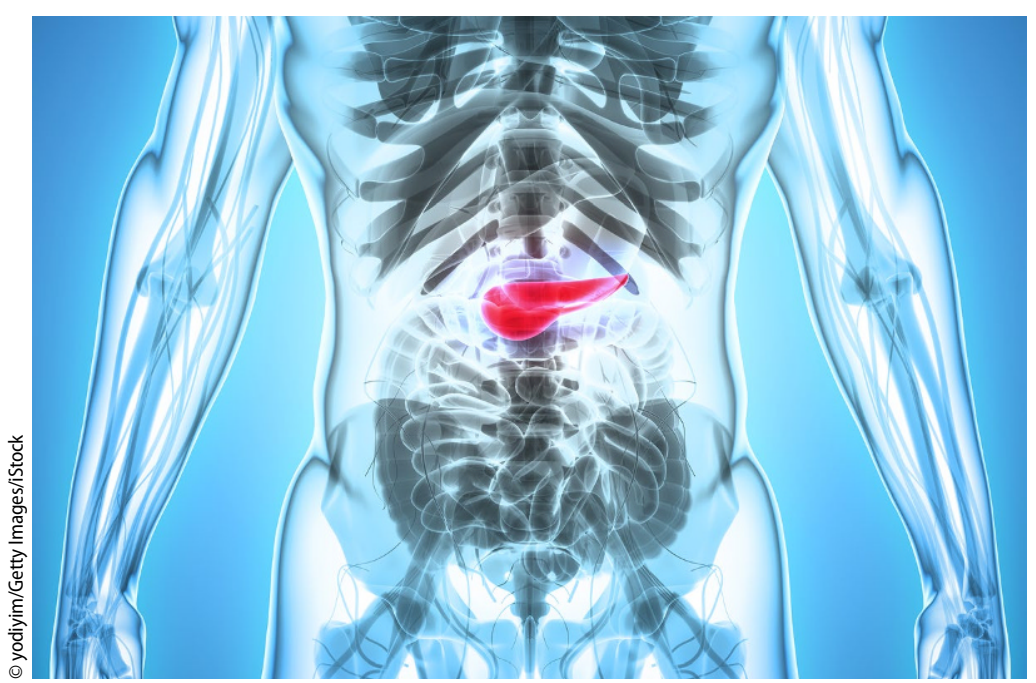

von Nervenzellen. Die Zugabe von Extrakten aus den Patientengewebeproben zu Nervenzellkulturen erhöhte die Menge des Enzyms nNOS in den Nervenzellen.

\section{Im Mausmodell getestet}

In einem Mausmodell für Pankreaserkrankungen setzten Demir und Kollegen anschließend einen spezifischen Hemmstoff ein, der nNOS blockiert. Dieser Stoff ist bereits experimentell zugelassen, darf aber noch nicht beim Menschen eingesetzt werden. Mäuse, die den Wirkstoff erhielten, reagierten jedenfalls weniger empfindlich auf Berührungen im betroffenen Bauchbereich als die Kontrolltiere. Weitere Tests des neuen Wirkstoffs, sollen das Potenzial für einen möglichen künftigen Einsatz als alternative Schmerztherapie für Patienten mit Krankheiten des Pankreas untersuchen.

$E b$

Wien klin Mag 2020 · 23:25

https://doi.org/10.1007/s00740-020-

00327-3

Online publiziert: 14. Januar 2020

(c) Springer-Verlag GmbH Austria, ein Teil von Springer Nature 2020 